\title{
A Synergistic Approach to the Real-Time Estimation of the Feet Ground Reaction Forces and Centers of Pressure in Humans With Application to Human-Robot Collaboration
}

\author{
Marta Lorenzini ${ }^{\circledR}$, Wansoo Kim ${ }^{\circledR}$, Elena De Momi ${ }^{\circledR}$, and Arash Ajoudani ${ }^{(1)}$
}

\begin{abstract}
This letter proposes a novel technique for the realtime estimation of the human feet ground reaction forces (GRFs) and centers of pressure (CoP) from the whole-body CoP and body configuration. The estimated variables are used for the estimation of the overloading torques in human joints during double support, with the aim to provide an evaluation of the human ergonomics while performing heavy manipulation tasks with a robot, or when exposed to an external force. The estimation of the feet GRFs and CoP is achieved using a synergistic approach that combines a simplified geometrical model of the whole-body CoP and a learning technique to address the underdetermined force distribution problem. First, a statically equivalent serial chain (SESC) model which enables the whole-body CoP estimation is identified. Then, the estimated whole-body CoP and the simplified body pose information are used for the training and validation of the learning technique. The displacements between the feet $\mathrm{CoP}$ estimated using the synergistic model and the values measured using insole sensors, along with the GRFs vectors are finally used for the estimation of the body overloading joint torques. The proposed synergistic model is first validated experimentally in five subjects. Next, its real-time efficacy is assessed in a human-robot load sharing task, in which the robot trajectories are optimized to minimize the effect of the external load on the human joints.
\end{abstract}

Index Terms-Human-centered robotics, human factors and human-in-the-loop, physical human-robot interaction.

\section{INTRODUCTION}

I $\mathrm{N}$ THE last few years, the great potential and the countless benefits of human-robot collaboration (HRC) are becoming increasingly evident among the scientific and industrial communities. The robots can reduce fatigue and stress by

Manuscript received February 24, 2018; accepted July 1, 2018. Date of publication July 13, 2018; date of current version August 2, 2018. This letter was recommended for publication by Associate Editor C. Secchi and Editor Y. Yokokohji upon evaluation of the reviewers' comments. (Marta Lorenzini and Wansoo Kim contributed equally to this work.) (Corresponding author: Marta Lorenzini.)

M. Lorenzini is with the Human-Robot Interaction Lab, Department of Advanced Robotics, Istituto Italiano di Tecnologia, Genoa 16163, Italy, and also with the Department of Electronics, Information and Bioengineering, Politecnico di Milano, Milano 20133, Italy (e-mail: marta.lorenzini@iit.it).

W. Kim and A. Ajoudani are with the Human-Robot Interaction Lab, Department of Advanced Robotics, Istituto Italiano di Tecnologia, Genoa 16163, Italy (e-mail: wan-soo.kim@iit.it; arash.ajoudani@iit.it).

E. De Momi is with the Department of Electronics, Information and Bioengineering, Politecnico di Milano, Milano 20133, Italy (e-mail: elena.demomi@ polimi.it).

Digital Object Identifier 10.1109/LRA.2018.2855802 providing assistance, increase human capabilities while preventing injuries in industrial scenarios, automatize therapies in rehabilitation contexts, and improve in general the quality of life [1]. On the other hand, humans can provide experience, transfer knowledge and supervise robots functionalities, adding a certain level of flexibility to the process and contributing to an effective accomplishment of a broad range of tasks [2].

To successfully introduce robotic systems into human environments, safety considerations are among the key factors to be addressed [3]. Collision avoidance [4], [5] and impact force control approaches [6], [7], are among the most common strategies to guarantee safety in HRC, due to the underlying physical interactions between the humans and the robots [8]. Nevertheless, it is worth noticing that musculoskeletal disorders (MSD) are the single largest category of work-related diseases in many industrial countries [9], and impose extremely large costs each year in lost productivity and absence due to sickness. One of the greatest contributors to MSDs in every industry division is repetitive manipulation or carrying of heavy objects, due to the mechanical overloading of body joints [10], [11]. The most affected body part is the trunk, but numerous injuries to upper and lower extremities have also been reported [12].

With the purpose of mitigating potential injuries or chronic pathology risks during the HRC tasks, in our previous work [13] we developed a framework to monitor human joint torque variations in real-time during the collaborative tasks. The proposed method was based on the real-time estimation of the translational displacement of the whole body centers of pressure $(\mathrm{CoP})$ in the presence of external forces, used along with the ground reaction forces (GRFs) vectors to compute and monitor the overloading joint torques throughout the task execution. This information was used for the on-line optimisation of the robot trajectories to assist the human partner in reaching more ergonomic and comfortable configurations of the body. The human subject was also provided with a visual feedback to help him/her to achieve the most suitable body configuration but the information displayed were limited.

The real-time CoP model employed in this procedure was constructed and identified by a statically equivalent serial chain (SESC) technique [14], which uses body pose measurements from a suitable motion-capture system. Since this method allows solely to obtain the whole body CoP, the HRC technique 


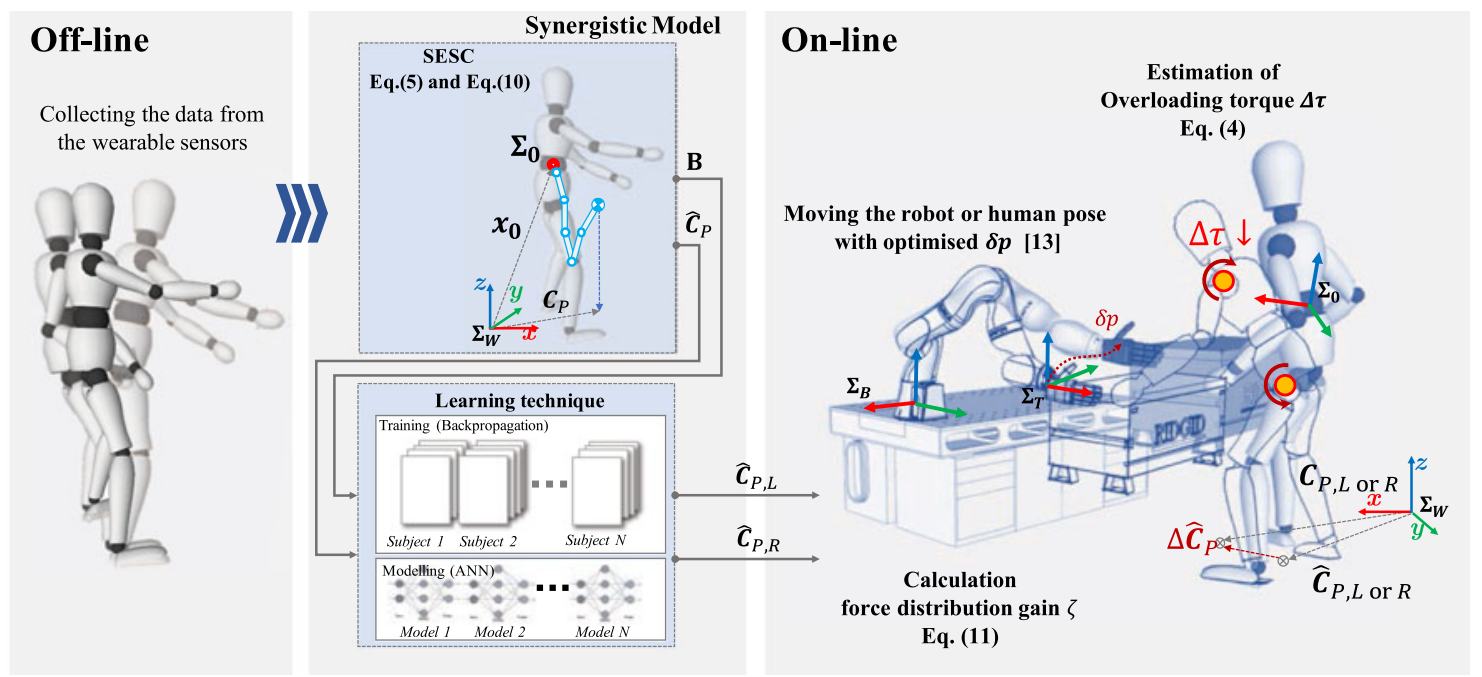

Fig. 1. The overall procedure for estimating the feet GRFs and CoP using the synergistic model (left), and reducing the overloading joint torque during HRC in the on-line phase (right).

proposed in [13] can currently be applied to human biomechanical models represented by planar serial chains that include just one GRF. The development of this reduced model to a three dimensional (3D) one including both legs, and thus the contact forces exchanged by both feet with the ground, would enable the tracking of human states in a larger workspace and broaden the applications of this technique to more realistic and complex 3D HRC tasks. To this end, a method to estimate the feet CoP and GRFs, during double support, on the basis of the whole body CoP and the total GRF is needed. Nevertheless, the computation of physically feasible contact forces to match a desired net contact wrench, known as the force distribution problem (FDP), is known to be an under-determined problem.

In robotics community, solutions to this problem have been addressed to properly select foot forces in multi-legged robots with a view to simplification, while assuming point contacts at the feet which can generate no moments [15], [16]. For humanoid robots, due to planar contacts which can generate significant moments, this assumption is not allowed and a more general distribution of the wrench to each foot is required. Although this complexity introduces additional constraints on the individual foot wrenches, the FDP is generally under-specified and provides the opportunity to optimise the feet $\mathrm{CoP}$ and contact forces. Several studies have been focused on the use of constrained optimisation methods which employ sub-optimal solutions by applying simplifying assumptions [17], [18]. All these approaches, in general, select a specific objective - as the tangential force [19], contact force norms [20], ground force magnitudes [17], and ankle torques [18] - on the basis of the context or the application for which the methods are developed and then minimise the corresponding objective function. This kind of formulation can provide quite good results in the robotic domain but it is not suitable for humans. The way in which a task should be performed by a robot is decided a priori and thus adding equations that correspond to selected constraints - with the aim to solve the un-determinacy of the FDP - is quite simple. Determining in advance which strategy a human subject is going to use to perform a task, instead, it is not so straightforward.
Accordingly, the aim of this letter is to propose a novel approach to solve the un-determinacy of the FDP for the human. Unlike task-specific robotics solutions, here we build a relation that links the whole body GRF and CoP to the feet GRFs and $\mathrm{CoP}$, directly based on the data measured on the human subjects. We present a synergistic method which combines the information enclosed in the SESC model for the whole-body CoP estimation with the ability of learning techniques to implicitly detect complex nonlinear relationships between variables.

The overall procedure of our approach is shown in Fig. 1 and can be divided into two main parts: the off-line and the on-line phases. In the off-line phase we build a large dataset which allows us to characterize the method. Using external sensory systems, we collect sets of measurements of the human whole body pose along with CoP and GRFs upon a large number of static postures. Initially, this dataset is used to identify the unknown body segment inertial parameters (BSIPs) of the human body required for the development of the CoP model with the SESC technique. Secondly, to design and train a feed-forward artificial neural network (ANN) employed for solving the FDP. The proposed synergistic model is experimentally evaluated on five subjects. Then, in the on-line phase, its real-time applicability is experimentally evaluated. The overloading joint torques of the human body are estimated in the double support phase using a reduced-complexity biomechanical model while executing a load-sharing task with a robot. Consequently, robot trajectories are optimised to minimise such overloading torques, by considering the human, the robot and the task constraints. Being helped by the robot and by following a visual feedback interface that we have implemented, the human subject should then change his/her body configuration to achieve a more ergonomic and comfortable one.

\section{WEARABLE SENSORY SYSTEMS}

This section introduces the wearable sensory systems in our experimental setup, as illustrated in Fig. 2. The use of wearable and light-weight sensory systems for the estimation of the 


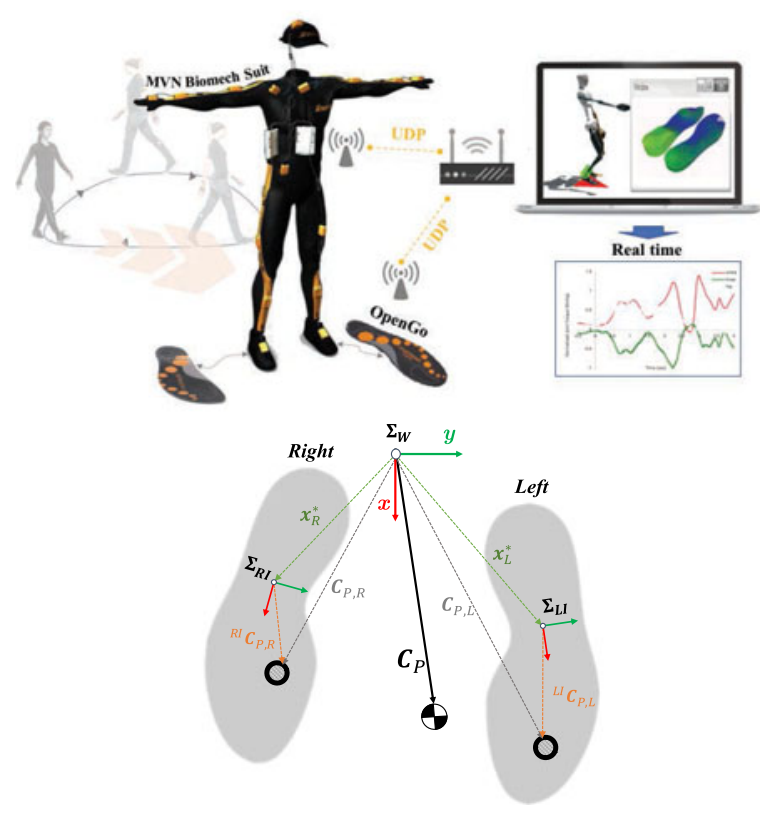

Fig. 2. The selected wearable sensor systems (a MoCap suit and insole sensors) and the corresponding frames for the $\mathrm{CoP}$ in this study are illustrated.

human dynamic states in HRC scenarios is of crucial importance since such systems do not add additional constraints on the human mobility. The measurement of the whole-body human motion is achieved using a wearable MVN Biomech suit (Xsens Tech) provided with seventeen inter-connected inertial measurement unit (IMU) sensors. In comparison to the multicamera systems that suffer from marker visibility issues due to the occlusions and the limited range of camera visibility, the wireless motion capture (MoCap) system exploited in this study enables the user to naturally perform the tasks in a large workspace. The on-line streaming of the human joints and segments data is performed using the MVN Biomech software. The calculation of the CoP and the measurement of the vertical GRF (vGRF) in this study are performed using OpenGo insole sensors (Moticon $\mathrm{GmbH}$ ). In comparison to the force plate system that can severely reduce the subject's mobility, insole sensors are easily wearable (by placing them inside the shoes) and do not impose any constraints on the wearer's mobility. The OpenGo insole sensor device consists of thirteen capacitive pressure sensing pads and a 3D accelerometer for measuring motion. Furthermore, to improve the reliability of sensing capability, we developed a supplementary mechanical part with a thin and flexible surface as an additional attachment part to the OpenGo. The designed mechanical part has protuberances on the lower surface that are placed at the centers of the capacitive pressure sensing pads, enabling an effective distribution of body weight over the insole sensors. This consideration contributes to a significant increase of the feet GRFs and CoP measurement accuracy. The acquisition, synchronization and processing of the sensory data are achieved using an external PC in C++ environment. Both sensory units (MoCap and insole sensor system) can provide the required information in real-time over a wireless connection with the external PC.

\section{OVERLOADING JOINT TORQUES}

We recently proposed an algorithm to account for the human overloading joint torques ${ }^{1}$ in real-time [21]. A brief summary of the method, with extension to the double support phase, is presented here. The method is based on the displacements of the $\mathrm{CoP}$, calculated from the difference between an estimated one (using an off-line calibrated model) and the measured one (using the wearable sensors) as mentioned in the previous Section II. If no external interactions of the human with the environment (or object) are in place, the estimation of the CoP vector $\hat{\mathbf{C}}_{P_{w} \text { o }}$ achieved by the human body model is similar to the measured one $\mathbf{C}_{P_{w t}}$. If an external force is applied to the human, the estimated and the measured CoP vectors differ. Accordingly, the overloading joint torque vector is estimated from the CoP displacement vector and calculated from the difference between the estimated one $\hat{\mathbf{C}}_{P_{w o}}$ and the measured one $\mathbf{C}_{P_{w t}}$.

The human model in this letter is based on a floating base. The pelvis frame is chosen as the base frame $\Sigma_{0}$ attached to the inertial frame $\Sigma_{W}$ through six virtual degrees of freedom (DoFs). The generalised coordinates of the system are defined by $\mathbf{q}=\left[\begin{array}{lll}\mathbf{x}_{0}^{T} & \boldsymbol{\theta}_{0}^{T} & \mathbf{q}_{h}^{T}\end{array}\right]^{T} \in \mathbb{R}^{6+n} \cdot \mathbf{x}_{0}^{T}$ and $\boldsymbol{\theta}_{0}^{T}$ represent the position and the orientation of $\Sigma_{0}$, respectively. The angular position of human joints is denoted by $\mathbf{q}_{h}=\left[\begin{array}{lll}q_{1} & \cdots & q_{n}\end{array}\right]^{T} \in \mathbb{R}^{n}$ where $n$ is the number of joints. $\mathbf{q}$ as well as the actual CoP vector $\mathbf{C}_{P_{w t}}$ can be measured by the MVN Biomech suit and the OpenGo insole sensor system, respectively.

The human joint torque vector $\boldsymbol{\tau}_{w o}$ with no external load except the body weight can be calculated using $\hat{\mathbf{C}}_{P_{w o}}$ and the vGRF vector $\mathbf{f}_{w o}$ as follows:

$$
\mathbf{S}^{T} \boldsymbol{\tau}_{w o}=\boldsymbol{\tau}_{b}-\sum_{i=1}^{n_{f}} \mathbf{J}_{\hat{C}_{P w o}}(\mathbf{q})^{T} \mathbf{f}_{w o, i} .
$$

Similarly, we calculate the torque vector $\tau_{w t}$ in an externally loaded condition by using $\mathbf{C}_{P_{w t}}$ and the vGRF vector $\mathbf{f}_{w t}$ as follows:

$$
\mathbf{S}^{T} \boldsymbol{\tau}_{w t}=\boldsymbol{\tau}_{b}-\sum_{i=1}^{n_{f}} \mathbf{J}_{C_{P_{w} i}}(\mathbf{q})^{T} \mathbf{f}_{w t, i}-\sum_{j=1}^{n_{h}} \mathbf{J}_{a_{h j}}(\mathbf{q})^{T} \mathbf{f}_{h, j},
$$

where $\boldsymbol{\tau}_{b} \in \mathbb{R}^{n+6}$ is equal to $\mathbf{M}(\mathbf{q}) \ddot{\mathbf{q}}+\mathbf{C}(\mathbf{q}, \dot{\mathbf{q}}) \dot{\mathbf{q}}+\mathbf{G}(\mathbf{q}) \in$ $\mathbb{R}^{n+6}$, where $\mathbf{M}, \mathbf{C}$ and $\mathbf{G}$ represent the inertia matrix, the vector of centrifugal and Coriolis forces, and the vector of the gravity force, respectively. $n_{f}$ is the number of contact forces exchanged with the ground. $\mathbf{f}_{w o}$ and $\mathbf{f}_{w t}$ are the vGRF vectors applied at the CoP of each foot without and with the effect of external forces, which can be considered as the body weight of human and the one measured by sensors, respectively. $\mathbf{f}_{h}$ represents the interaction forces that are applied at the contact points $\mathbf{a}_{h}$, excluding the feet contacts. $n_{h}$ is the number of contact points where the external forces are applied. In addition, $\mathbf{S}=\left[\begin{array}{ll}\mathbf{0}_{n \times 6} & \mathbf{I}_{n \times n}\end{array}\right] \in \mathbb{R}^{n \times(n+6)}$ is the actuation matrix and $\mathbf{J}_{p_{i}}(\mathbf{q})$ is the contact Jacobian at the point $\mathbf{p}_{i}$ (e.g. positions of the feet CoP, and arm endpoints) with respect to $\Sigma_{W}$.

\footnotetext{
${ }^{1}$ The overloading joint torque refers to the torque induced into the human joint by an external load.
} 
The relationship between the interaction force $\mathbf{f}_{h}$ and vGRF variation $\Delta \mathbf{f}_{w, i}=\mathbf{f}_{w t, i}-\mathbf{f}_{w o, i}$ can be defined as

$$
\Delta \mathbf{F}=\sum_{i=1}^{n_{f}} \Delta \mathbf{f}_{w, i}=-\sum_{j=1}^{n_{h}} \mathbf{f}_{h, j}
$$

Deriving from (1), (2) and (3), the overloading joint torques can be defined as

$$
\begin{aligned}
\Delta \boldsymbol{\tau}_{s}= & \mathbf{S}^{T}\left(\boldsymbol{\tau}_{w t}-\boldsymbol{\tau}_{w o}\right) \\
= & \sum_{j=1}^{n_{h}} \mathbf{J}_{a_{h j}}(\mathbf{q})^{T} \eta_{j} \Delta \mathbf{F} \\
& -\sum_{i=1}^{n_{f}}\left(\mathbf{J}_{\Delta C_{P i}}(\mathbf{q})^{T} \mathbf{f}_{w t, i}+\mathbf{J}_{\hat{C}_{P w o i}}(\mathbf{q})^{T} \zeta_{i} \Delta \mathbf{F}\right),
\end{aligned}
$$

with the Jacobian of the CoP displacement defined as $\mathbf{J}_{\Delta C_{P i}}=$ $\mathbf{J}_{C_{P_{w} i}}-\mathbf{J}_{\hat{C}_{P w o i}}$. It is important to note that $\boldsymbol{\tau}_{b}$ does not affect the overloading joint torque vector $\Delta \tau_{s}$ in any body configuration since the external load is included in $\Delta \tau_{s}$ itself. In the meantime, this consideration significantly reduces the number of human model parameters to be identified [22]. $0 \leq \zeta_{i}, \eta_{j} \leq 1$ are the distribution gains for vGRF and interaction forces, respectively, which can be calculated from the body configuration [23], [24] (N.B. $\sum_{i} \zeta_{i}=1, \sum_{j} \eta_{j}=1$ is a necessary condition). In the next section, we provide a method to estimate the approximate distribution gains from the feet CoP positions.

\section{SYNERGISTIC MODEL}

In this section, we introduce a novel synergistic model (see in the middle of Fig. 1) that combines a simplified geometrical model and a learning technique for solving the FDP and thus estimating the feet GRFs and CoP in real-time. Once it has been identified and trained in the off-line phase, the model can be then employed in the algorithm to calculate the overloading joint torques in the on-line phase, as mentioned in the previous section. Firstly, we identify the whole-body CoM model using the SESC technique, which is based on a reduced complexity dynamic modelling of the human subject and achieves an accuracy of the CoM estimation comparable to the accuracy reached by the classical models that are based on articulated chains [14], [25]. Since the SESC technique is a geometry-based reconstruction of the CoM, the observability of the CoM position is complete [26] and due to the principled simplification approach, the real-time compatibility is addressed [13]. The whole-body $\mathrm{CoP}$ can then be computed in the dynamic case. Next, the training of an ANN to map the whole-body CoP along with the body pose to feet CoP is performed. This consideration is to reduce the amount of modelling uncertainty that is expected to be learnt by the ANN.

\section{A. Geometrical Model: Whole-Body Center of Pressure}

The whole-body center of mass $(\mathrm{CoM}), \mathbf{C}_{M}=\left[C_{M x}\right.$ $\left.C_{M y} C_{M z}\right]^{T} \in \mathbb{R}^{3}$ of any branched chain (e.g., leg, arm, etc.) can be modelled by geometric parameters (i.e. CoM, mass and length of each link) of the original whole-body structure using the SESC technique [14]

$$
\mathbf{C}_{M}=\mathbf{x}_{0}+\mathbf{B} \boldsymbol{\Phi},
$$

where $\mathbf{x}_{0}$ is the position of $\Sigma_{0}$, matrix $\mathbf{B}=\left[\begin{array}{lll}\mathbf{A}_{0} & \cdots & \mathbf{A}_{n}\end{array}\right] \in$ $\mathbb{R}^{3 \times 3(n+1)}$ contains $i$-th link rotation matrices $\mathbf{A}_{i} \in S O(3)$ with respect to $\Sigma_{W}$. Matrix $\boldsymbol{\Phi}=\left[\begin{array}{lll}\boldsymbol{\phi}_{0}^{T} & \cdots & \boldsymbol{\phi}_{n}^{T}\end{array}\right]^{T} \in \mathbb{R}^{3(n+1)}$ includes the vector of SESC parameters $\phi_{i} \in \mathbb{R}^{3}$, which refers to mass distribution of the human model.

To identify the unknown parameters, the whole-body CoM can be written in regressor form as

$$
{ }^{0} \mathbf{C}_{M}=\mathbf{C}_{M}-\mathbf{x}_{0}=\mathbf{B} \Phi,
$$

where ${ }^{0} \mathbf{C}_{M}$ is the CoM represented in $\Sigma_{0}$. The regression matrix $\mathbf{B}$ and the parameter vector $\boldsymbol{\Phi}$ contain all the known and unknown parameters of the SESC.

The identification of the parameter vector $\boldsymbol{\Phi}$ in such a form can be considered as a classical least-squares problem. In this study, the rotation matrix $\mathbf{B}$ and the human base frame vector $\mathbf{x}_{0}$ are calculated from the MVN Biomech suit measurements. On the other hand, the CoM vector cannot be obtained directly from the sensors but it is possible to achieve the ground-projected $\mathrm{CoM}$, which corresponds to the $\mathrm{CoP}$ in the static condition. The whole-body CoP vector $\mathbf{C}_{P}=\left[C_{P x} C_{P y}\right]^{T} \in \mathbb{R}^{2}$ with respect to the $\Sigma_{W}$ can thus be calculated using the OpenGo insole sensor system as

$$
\mathbf{C}_{P}=\frac{\mathbf{f}_{L} \cdot \mathbf{C}_{P, L}+\mathbf{f}_{R} \cdot \mathbf{C}_{P, R}}{\mathbf{f}_{L}+\mathbf{f}_{R}},
$$

where, $\mathbf{f}_{L}$ and $\mathbf{f}_{R}$ are the GRF of left and right foot, respectively. The CoP of each foot $\mathbf{C}_{P, L}$ and $\mathbf{C}_{P, R}$ with respect to the $\Sigma_{W}$ can be calculated as follows

$$
\mathbf{C}_{P, L}=\mathbf{x}_{L}^{*}+\mathbf{A}_{L}^{* L I} \mathbf{C}_{P, L}, \text { and } \mathbf{C}_{P, R}=\mathbf{x}_{R}^{*}+\mathbf{A}_{R}^{* R I} \mathbf{C}_{P, R} .
$$

The superscript $(.)^{*}$ above symbolises the pre-multiplication of the projection to the $x-y$ ground plane. The position of the feet $\mathbf{x}_{L}^{*}$ and $\mathbf{x}_{R}^{*} \in \mathbb{R}^{2}$, and the corresponding orientations $\mathbf{A}_{L}^{*}$ and $\mathbf{A}_{R}^{*} \in \mathbb{R}^{2 \times 2}$ are measured from the MVN Biomech suit. ${ }^{L I} \mathbf{C}_{P, L}$ and ${ }^{R I} \mathbf{C}_{P, R}$ correspond to the measured CoP value with respect to the insole sensor frame on left and right, respectively. Accordingly, the least-squares problem can be solved by the stacked matrices for $p$ pose set of $\mathbf{B}^{*}$ and ${ }^{0} \mathbf{C}_{P}$ as $\mathbf{W} \in \mathbb{R}^{2 p \times 3(n+1)}$ and $\Omega \in \mathbb{R}^{2 p \times 1}$, respectively. The vector of the identified SESC parameters $\hat{\boldsymbol{\Phi}} \in \mathbb{R}^{3(n+1)}$ can be calculated as

$$
\hat{\boldsymbol{\Phi}}=\mathbf{W}^{+} \boldsymbol{\Omega},
$$

where $\mathbf{W}^{+}=\left(\mathbf{W}^{T} \mathbf{W}\right)^{-1} \mathbf{W}^{T}$ is the Moore-Penrose generalised inverse.

In the static condition, we can compute the CoP by projecting the whole-body CoM estimated by (5) with the identified SESC parameters (9) onto the $x-y$ plane. On the other hand, the position of the $\mathrm{CoP}$ in the dynamic condition, relative to the CoM, can be computed by the differences between the acceleration about the CoM and the angular momentum [27], [28]. Nevertheless, for a whole-body resting on a flat ground surface and rotationally stable, the angular momentum variations of the $\mathrm{CoM}$ are negligible. Thus, we can obtain the estimated CoP 
vector $\hat{\mathbf{C}}_{P}$ on the contact surface in the dynamic condition as

$$
\hat{\mathbf{C}}_{P}=\left[\begin{array}{c}
\hat{C}_{P x} \\
\hat{C}_{P y}
\end{array}\right]=\left[\begin{array}{l}
\hat{C}_{M x} \\
\hat{C}_{M y}
\end{array}\right]-\frac{\hat{C}_{M z}}{\ddot{\hat{C}}_{M z}+g}\left[\begin{array}{c}
\ddot{\hat{C}}_{M x} \\
\ddot{\hat{C}}_{M y}
\end{array}\right],
$$

where $g$ is gravitational constant, $\hat{\mathbf{C}}_{M}$ is the estimated CoM vector from the SESC, and $\ddot{\hat{C}}_{M x}, \ddot{\hat{C}}_{M y}$ and $\ddot{\hat{C}}_{M z}$ are the linear acceleration of the CoM. Consequently, we only consider the second derivative of the CoM vector and $g$ in order to determine the $\mathrm{CoP}$ of the human body. In addition, the acceleration of the CoM vector is calculated with the Kalman filtering approach [29].

\section{B. Learning Technique: Feet Centers of Pressure}

Since the main contribution of this letter is to manage the double support indeterminacy and thus the case in which $n_{f}$ is equal to 2 in (4), we employ the multi-layer ANN technique to estimate the feet GRFs and CoP, using as inputs the whole-body CoP estimated by the simplified SESC model and body configurations measured with the MVN Biomech suit. Supporting this choice, it has been shown that supervised multi-layer ANN with the proper input data and a non-linear activation functions are capable of representing accurate approximations and mappings [30]. Specifically, a feed-forward ANN with one hidden layer and enough number of neurons in the hidden layers can fit any finite input-output mapping problem [31].

The training of the ANN model for each subject is performed in the off-line phase so as to build the real-time model. To achieve the best results possible, different combinations of training functions, numbers of neurons in the hidden layer and sets of input data are tested. As regards the input data, the wholebody $\hat{\mathbf{C}}_{P}$ from the SESC model, the orientation matrix of the simplified human model $\mathbf{B}$ and the pelvis, the left foot and the right foot positions are ultimately employed for the training and the validation. As regards the structure, we build a feed-forward ANN composed of one hidden layer containing four neurons with a non-linear activation function and an output layer with a linear function. The network is trained with the target data (e.g. the measured feet CoP using the OpenGo insole sensor) using the weights and bias values according to Levenberg-Marquardt optimisation.

Consequently, by using the proposed synergistic model, the feet $\operatorname{CoP} \hat{\mathbf{C}}_{P, R}$ and $\hat{\mathbf{C}}_{P, L}$ can be estimated in real-time. In addition, we can obtain, deriving from (7), the distribution gain $\zeta$ for each foot as

$$
\left[\begin{array}{ll}
\zeta_{L} & \zeta_{R}
\end{array}\right]=\left[\left|\frac{\hat{\mathbf{C}}_{P}-\hat{\mathbf{C}}_{P, R}}{\hat{\mathbf{C}}_{P, L}-\hat{\mathbf{C}}_{P, R}}\right|\left|\frac{\hat{\mathbf{C}}_{P}-\hat{\mathbf{C}}_{P, L}}{\hat{\mathbf{C}}_{P, R}-\hat{\mathbf{C}}_{P, L}}\right|\right] .
$$

Reminding that $\sum_{i} \zeta_{i}=1$ it is possible to compute the gain related to one foot and then subtract its value from 1 to obtain the gain related to the other foot.

\section{VerificATION OF THE METHOD}

This section first provides the performance evaluation of the proposed synergistic model. Next, a human-robot load sharing task is performed to validate the real-time applicability of the
TABLE I

Means and Standard ERrors of the Position ERrors BetweEn the Whole Body CoP MeAsured Using THE OpenGo Insole SENSORS, AND THE COP EsTIMATED BY THE IDENTIFIED SESC MODEL. THE ERRORS ARE COMPUTED FOR EACH SUBJECT ON THE X-AXIS AND ON THE Y-AXIS ACROSS 200 POSTURES

\begin{tabular}{ccc}
\multirow{2}{*}{ Subject } & \multicolumn{2}{c}{ CoP errors $(\mathrm{m})$} \\
\cline { 2 - 3 } & $C_{P_{x}, L}$ & $C_{P_{y}, L}$ \\
\hline 1 & $1.34^{*} \pm 0.04^{*}$ & $1.33^{*} \pm 0.04^{*}$ \\
2 & $1.57^{*} \pm 0.04^{*}$ & $1.34^{*} \pm 0.04^{*}$ \\
3 & $1.45^{*} \pm 0.04^{*}$ & $1.56^{*} \pm 0.04^{*}$ \\
4 & $1.76^{*} \pm 0.05^{*}$ & $1.70^{*} \pm 0.05^{*}$ \\
5 & $1.83^{*} \pm 0.05^{*}$ & $1.40^{*} \pm 0.05^{*}$ \\
\hline
\end{tabular}

*All the values in the table are multiplied by $10^{-2}$.

method. The objective of the HRC task is to minimise the human overloading joint torques computed in real-time by means of the synergistic model and to achieve anticipatory optimisation of robot behaviour to promote ergonomic HRC.

\section{A. Model Identification and Validation}

Five healthy male volunteers (age: $28.6 \pm 4.3$ years; mass: $84.7 \pm 10.7 \mathrm{~kg}$; height: $182.2 \pm 2.9 \mathrm{~cm})^{2}$ were recruited in the experimental session. A written informed consent was obtained after explaining the experimental procedure. Each subject was asked to wear the MVN Biomech suit and the OpenGo insole sensors and then required to hold 200 static poses for the data collection. During the acquisition, the postures were chosen by each subject arbitrarily but with the requirement to change the orientations of each segment and the position of the feet CoP as much as possible in between, to obtain variables as linearlyindependent as possible. A large number of postures collected along with their variability are necessary to build a suitable set of input data for the parameters identification of the proposed synergistic model.

The SESC parameters identification was done using 80 selected static poses that were considered suitable given the level of approximation of our human model (details can be found in [14]). Table I presents the means and the standard errors of the position error of the CoP with respect to the $\Sigma_{W}$ to evaluate the performance of the SESC technique. This position error was computed in $x$ - and in $y$-direction for all the 200 postures performed by five subjects. We compared $\mathbf{C}_{P_{w t}}$ and $\hat{\mathbf{C}}_{P_{w o} \text { po- }}$ sitions, computed with (7) using data measured by the OpenGo insole sensors and estimated by the SESC model, respectively, without the effect of external forces. The mean position error was $1.83 \times 10^{-2} \mathrm{~m}$ in the $\mathrm{x}$-direction and $1.40 \times 10^{-2} \mathrm{~m}$ in the $y$-direction across all postures for the subject with the worst results, providing a solid evidence on the accuracy of the on-line CoP model. For the training of the ANN we used 160 static poses (around $80 \%$ of the whole dataset) which were in fact adopted as the training set.

Fig. 3 is a graphical representation of the means and the standard errors of the position errors between the feet $\mathrm{CoP}$ with respect to the $\Sigma_{W}$, measured by the OpenGo insole sensors and the estimated ones by the ANN, hence the output of the synergistic method. These errors were computed for each subject on

\footnotetext{
${ }^{2}$ Subject data is reported as: mean \pm standard deviation.
} 

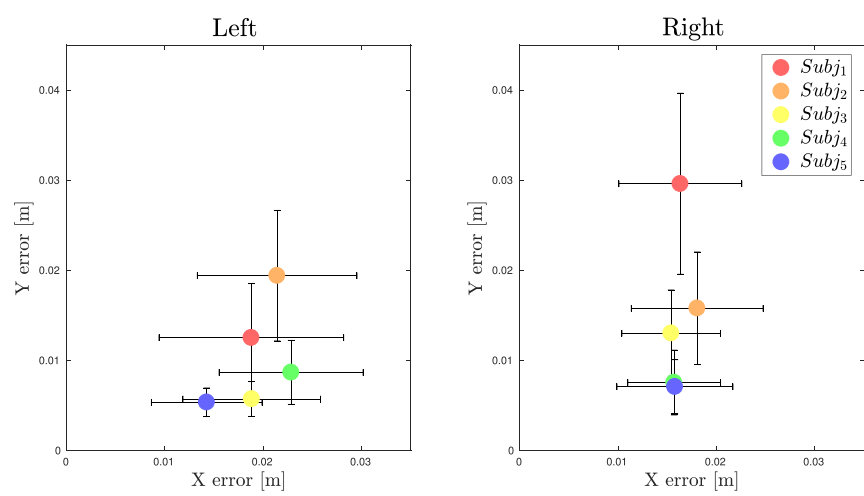

Fig. 3. Graphical representation of the means and standard errors of the position errors for the left foot (left chart) and for the right foot (right chart) between the feet $\mathrm{CoP}$ measured using the OpenGo insole sensors and the feet $\mathrm{CoP}$ estimated by the synergistic method. The feet CoP position errors are expressed in the inertial frame and computed for each subject on the $\mathrm{x}$-axis and on the $\mathrm{y}$-axis across 40 postures.

the $\mathrm{x}$-axis and on the $\mathrm{y}$-axis for the left foot and for the right foot across 40 postures that were used as the validation set to examine the network performance (around the other $20 \%$ of the whole dataset). The magnitude of the error was uniform between the subjects, demonstrating that the proposed synergistic approach can deal with varying patterns of movement and human body models with different inertial parameters. The consistency of the error in the $x$ and $y$ directions and the level of accuracy achieved were suitable for the target HRC task of this letter.

\section{B. Visual Feedback Interface}

In the human-robot collaboration experiment, the objective was to make the human subject perform the load sharing task in the most ergonomic way possible. To accomplish this requirement, the subject was supposed to change the body configuration by following two possible guidance: the assistance of the robot, whose functioning will be presented in the next paragraph, and a visual feedback. To this latter end, we took advantage of a graphic interface to make the subject aware of several meaningful information concerning his/her status. The ROS 3D visualizer RViz was employed for displaying purpose. The picture in the lower part of Fig. 4 highlights the data provided in real-time to the subject during the experiments: the current (black) and the optimal (red) (details about how it is computed can be found in the next paragraph) human body configurations, the degree of the computed overloading joint torques colour-coded to denote a high (red), medium (orange) or low (green) value and finally, the positions of measured and estimated feet CoP. To provide the model that estimated feet GRFs and CoP in real-time, we used the proposed synergistic approach, whose parameters were identified in the off-line phase. By following this visual feedback, displayed on a screen during the experiment, the subject could both monitor the level of the overloading torque on his/her own joint and change the current body configuration attempting to match the optimal one.

\section{Overloading Joint Torque Minimisation During HRC}

The second guidance provided to the human in the HRC task was the robot assistance. The robot must optimise the task

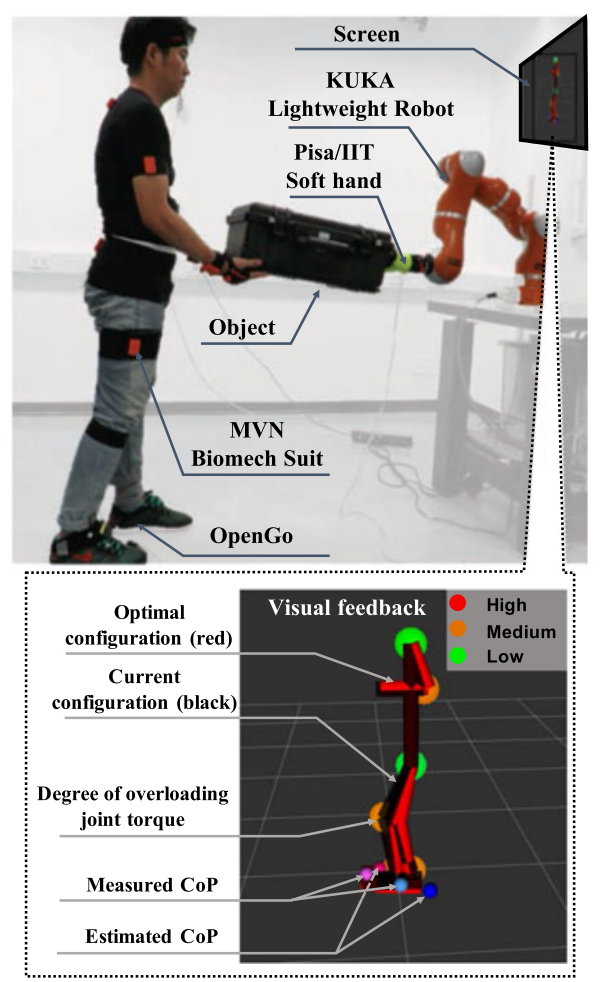

Fig. 4. Overview of the experimental setup: the photo on the top shows all the sensors and devices involved in the experiment, the picture on the bottom presents the visual feedback provided to the subject performing the experiment.

trajectories to minimise the human co-worker joints overloading, computed with equation (4), while performing the load sharing task. Fig. 4 shows the experimental setup. One subject (age: 30 years; mass: $76.5 \mathrm{~kg}$; and height: $1.78 \mathrm{~m}$ ) was asked to wear the MVN Biomech suit and the OpenGo insole sensors. The external object (width: $0.33 \mathrm{~m}$; depth: $0.56 \mathrm{~m}$; height: $0.23 \mathrm{~m}$; mass: $8 \mathrm{~kg}$ ) was grasped from two sides by the human hands and the Pisa/IIT Softhand [32] which was attached to a KUKA Lightweight robot (LWR). As the most co-manipulation scenarios, the considered task involves human hands to interact with an object, thus, we assume that the points of interaction with the external environments are located at the end of the forearm link and we assume a symmetric distribution ratio over them. Accordingly, the values of $\eta_{j}$ were set to 0.5 for both the left and right hand. The values of $\zeta_{j}$ are computed using (11).

In this experiment, the human subject had to achieve the human-robot load sharing task in a way that optimises the working conditions, including the constraints, in order to prevent any excessive load on the human joints. Accordingly, the human optimal body configuration was determined by an optimisation procedure based on the minimisation of the overloading joint torques with respect to body configuration and given constraints. Since the cost of optimisation should take into account the potential injuries arising from high overloading joint torques, we define a symmetric positive definite weight matrix $\mathbb{W}=\operatorname{diag}\left[\Delta \boldsymbol{\tau}_{1} / \boldsymbol{\tau}_{\max _{1}} \cdots \Delta \boldsymbol{\tau}_{n} / \boldsymbol{\tau}_{\max _{n}}\right] \in \mathbb{R}^{n \times n}$, with components $\tau_{\text {max }_{n}}$ that can be chosen from biomechanical data [33]. Given the weight matrix, the optimisation process is defined as

$$
\min _{\mathbf{q} h}\left\|\Delta \boldsymbol{\tau}^{T} \mathbb{W} \Delta \boldsymbol{\tau}\right\|,
$$


where $\mathbf{q}_{h} \in \mathbb{R}^{n}$ is the human joint angle vector and $\Delta \boldsymbol{\tau} \in \mathbb{R}^{n}$ is the vector of overloading joint torques that is calculated from (4). In the optimisation process, several constraints are considered: upper and lower bounds on the human joint angles $\mathbf{q}_{h}$, a linear inequality constraint on the $\operatorname{CoP} \mathbf{C}_{P} \in \mathbb{R}^{2}$ to keep the postural stability of the human body, constraints on the position of the object and finally a constraint for the endpoint manipulability of the human arm (details can be found in [13]). Once the human optimal body configuration was computed, the robot trajectory was adjusted accordingly to facilitate the subject to achieve more ergonomic poses. Consequently, during the HRC task, the human subject being helped by the robot as well as following the visual feedback, had to move the object and to change the body configuration so as to reach the optimal working conditions. Fig. 5 illustrates the results of HRC experiment. The first row depicts three specific configurations of the human biomechanical model while the subject is performing the task: the unoptimised (A), moving (B) and optimised (C) configurations, respectively. Observing the degree of the overloading joint torques represented by the colour-coded markers, it can be shown that, throughout the task, the external forces were redistributed among the joints so as to reach the human optimal configuration in phase (C). The fact that the elbow joint did not show the lowest level of joint overloading can be related to the condition imposed by the task of holding the object. The first plot presents the optimal trajectory that the robot perform to assist the subject during the task, both in time (left chart) and space (right chart). The second plot presents the computed overloading joint torques of the human: $\|\Delta \tau\|$ in the left hip $(\mathrm{LH})$, left knee (LK), left ankle (LA), right hip (RH), right knee (RK), right ankle (RA), elbow (E) and shoulder (S) joints. The overloading joint torques in the left and right arm joints had similar values because the arms moved symmetrically to hold the external object, thus the results for the right arm were demonstrated. As observed in the plot, starting from the initial configuration (A) to the optimised one (C), $\|\Delta \tau\|$ decreases in all joints due to the robot reactive behaviour, demonstrating that the effect of the external load on human joints was reduced. Specifically, the overall percentage of decrement was around $72.6 \%$ on average $(56.1 \%$ in the arms, $92.2 \%$ in the left leg and $35.3 \%$ in the right leg). ${ }^{3}$ Additionally, to compare the muscle activity in the whole-body through electromyography (EMG), we selected anterior deltoid (AD), biceps (BC), L5 paraspinals (L5), right and left vastus lateralis (R.VL and L.VL), right and left rectus femoris (R.RF and L.RF) and right and left tibialis anterior (R.TA and L.TA) muscles of the human counterpart. The reduction rates of the EMG signals (filtered and normalised) were computed and resulted as $70.9 \%$ in the $\mathrm{AD}, 8.6 \%$ in the $\mathrm{BC}$, $27.9 \%$ in $\mathrm{L} 5,62.3 \%$ on average for the right leg and $72.3 \%$ on average for the left leg, providing an additional evidence of the human effort attenuation. Finally, the lower plot of Fig 5 shows the displacements between the feet $\mathrm{CoP}$ measured by the insole sensors and the feet $\mathrm{CoP}$ estimated by the proposed synergistic

\footnotetext{
${ }^{3}$ Overloading torque reduction percentage in shoulder: $62.4 \%$, in elbows: $45.8 \%$, in left hip: $91.4 \%$, in left knee: $91.8 \%$, in left ankle: $93.4 \%$, in right hip $40.5 \%$, in right knee: $7.8 \%$, in right ankle: $43.5 \%$.
}
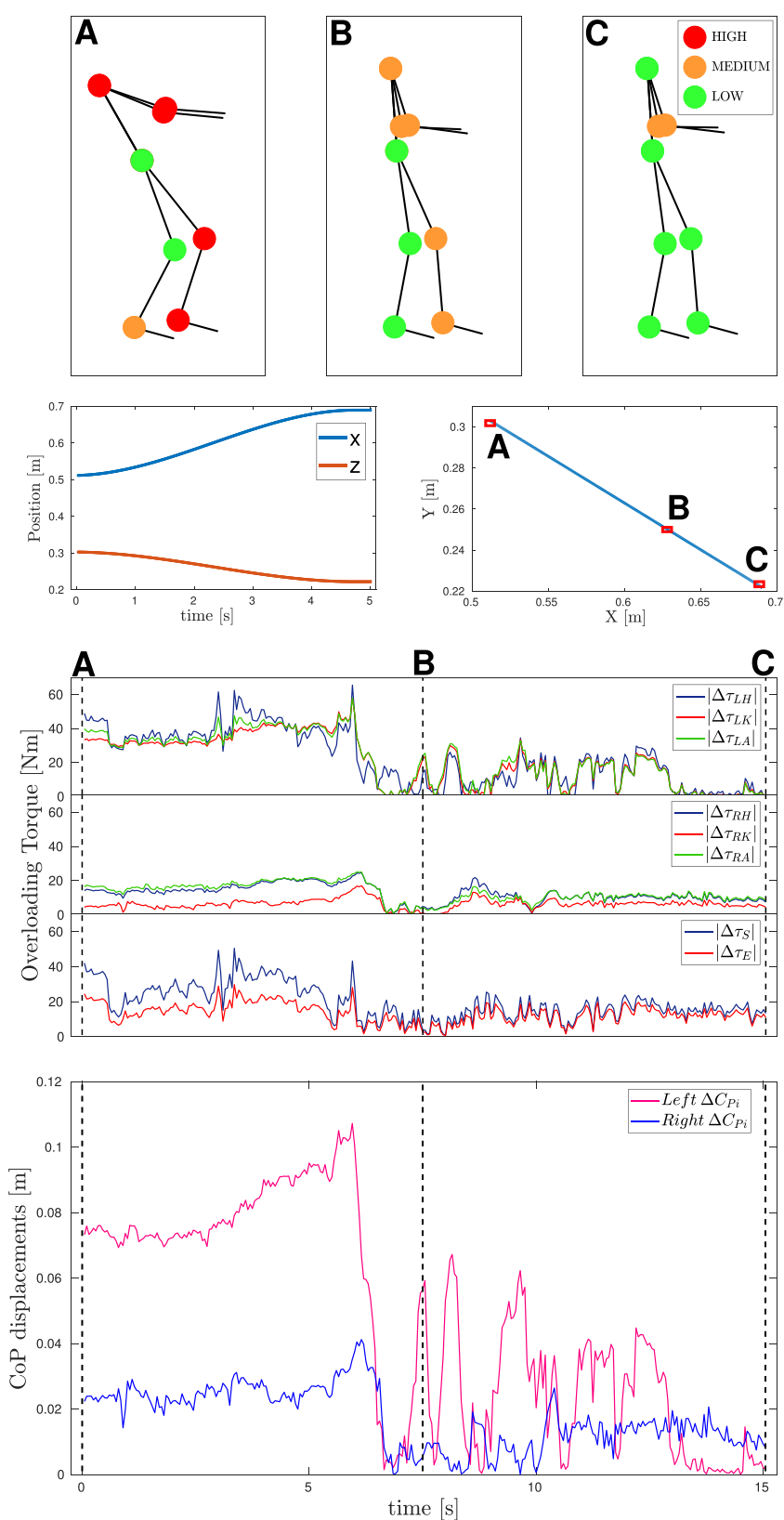

Fig. 5. Results of the human-robot load sharing task experiment. The sequence of the pictures on the top shows the progress of the experiment: unoptimised state (A), transition (B) and optimised state (C). The first plot shows the robot optimal trajectory both in space (left chart) and time (right chart). The second plot shows the calculated overloading joint torques. The third plot shows the displacements between the estimated and measured feet $\mathrm{CoP}$ for the right foot and for the left foot.

method. It was worth noticing that passing from configuration (A) to (C) this displacements decreases both for the right foot and for the left foot. This minimisation of the difference between $\mathbf{C}_{P}$ and $\hat{\mathbf{C}}_{P}$ provides a further evidence of the method capability to make the human achieve more ergonomic configurations.

\section{CONCLUSIONS AND FUTURE WORK}

In this work we proposed a synergistic approach for the real-time estimation of the human feet GRFs and CoP based on a reduced-complexity human body model and a learning 
technique, using wearable and light-weight sensory systems. The presented model was employed for the on-line estimation of the overloading joint torques in humans while performing a load sharing task with a robot. In comparison to our previous work [13], it enabled the extension of the HRC control framework to more realistic (including the double support phase) interaction scenarios. In addition, a visual feedback interface was developed using the ROS 3D visualizer RViz to make the subject aware of meaningful information regarding his/her status. The results proved that the proposed synergistic model is capable of solving the un-determinacy of the FDP for the human and provided promising evidences on its real-time applicability. Our choice to use a neural network to solve the FDP was due to the ability of such techniques to learn multi-input multioutput nonlinear systems, and to the fact that finding a nonlinear approximation by hand would not have been feasible considering the large input-output dimension. The training of several prototypes of neural networks with different numbers of layers attributed the best performance to the single-layer neural network. The optimisation execution time required roughly $1 \mathrm{~Hz}$ to be completed. Considering the task to be optimised (i.e., sharing the load), similar to our previous work [13], this latency did not affect the task performance and was acceptable to evaluate the presented model for double support.

Future works will focus on the improvement of the accuracy and reliability of the synergistic model by using dynamic data as the training set. Additionally, since the system of sensors employed in this work is quite complex and expensive, more practical and low-cost alternatives, e.g. vision systems embedded with a multi-person keypoint detection algorithm, will be considered to broaden the applicability of the framework. The proposed methodology has the potential to perform in dynamic cases, but this must be experimentally validated. Hence, more complex and articulated tasks including multiple serial passages and more dynamic and 3D movements of the human subject in the HRC scenario will be investigated.

\section{REFERENCES}

[1] A. Ajoudani, A. M. Zanchettin, S. Ivaldi, A. Albu-Schäffer, K. Kosuge, and O. Khatib, "Progress and prospects of the human-robot collaboration," Auton. Robots, vol. 42, pp. 957-975, Oct. 2017.

[2] O. Khatib, K. Yokoi, O. Brock, K. Chang, and A. Casal, "Robots in human environments: Basic autonomous capabilities," Int. J. Robot. Res., vol. 18, no. 7, pp. 684-696, 1999.

[3] R. Alami et al., "Safe and dependable physical human-robot interaction in anthropic domains: State of the art and challenges," in Proc. IEEE/RSJ Intell. Robots Syst., 2006, pp. 1-16.

[4] D. Kulić and E. Croft, "Pre-collision safety strategies for human-robot interaction," Auton. Robots, vol. 22, no. 2, pp. 149-164, 2007.

[5] C. Esteves, G. Arechavaleta, and J.-P. Laumond, "Motion planning for human-robot interaction in manipulation tasks," in Proc. IEEE Int. Conf. Mechatronics Autom., 2005, vol. 4. pp. 1766-1771.

[6] S. Haddadin et al., "On making robots understand safety: Embedding injury knowledge into control," Int. J. Robot. Res., vol. 31, no. 13, pp. 1578$1602,2012$.

[7] A. M. Zanchettin, N. M. Ceriani, P. Rocco, H. Ding, and B. Matthias, "Safety in human-robot collaborative manufacturing environments: Metrics and control," IEEE Trans. Automat. Sci. Eng., vol. 13, no. 2, pp. 882893, Apr. 2016.

[8] J. Kuehn and S. Haddadin, "An artificial robot nervous system to teach robots how to feel pain and reflexively react to potentially damaging contacts," IEEE Robot. Automat. Lett., vol. 2, no. 1, pp. 72-79, Jan. 2017.
[9] S. Bevan, T. Quadrello, R. McGee, M. Mahdon, A. Vavrovsky, and L. Barham, "Fit for work? musculoskeletal disorders in the european workforce. The work foundation," Sept. 2009. [Online]. Available: http://www.theworkfoundation.com/wf-reports/

[10] S. Kumar, "Theories of musculoskeletal injury causation," Ergonomics, vol. 44, no. 1, pp. 17-47, 2001.

[11] P. J. Carrivick, A. H. Lee, K. K. Yau, and M. R. Stevenson, "Evaluating the effectiveness of a participatory ergonomics approach in reducing the risk and severity of injuries from manual handling," Ergonomics, vol. 48, no. 8, pp. 907-914, 2005.

[12] J. Davies, G. Kemp, S. Frostick, C. Dickinson, and J. McElwaine, "Manual handling injuries and long term disability," Safety Sci., vol. 41, no. 7, pp. 611-625, 2003.

[13] W. Kim, J. Lee, L. Peternel, N. Tsagarakis, and A. Ajoudani, "Anticipatory robot assistance for the prevention of human static joint overloading in human-robot collaboration," IEEE Robot. Automat. Lett., vol. 3, no. 1, pp. 68-75, Jan. 2018.

[14] A. González, M. Hayashibe, V. Bonnet, and P. Fraisse, "Whole body center of mass estimation with portable sensors: Using the statically equivalent serial chain and a kinect," Sensors, vol. 14, no. 9, pp. 16 955-16 971, 2014.

[15] C. A. Klein and S. Kittivatcharapong, "Optimal force distribution for the legs of a walking machine with friction cone constraints," IEEE Trans. Robot. Automat., vol. 6, no. 1, pp. 73-85, Feb. 1990.

[16] F.-T. Cheng and D. E. Orin, "Optimal force distribution in multiple-chain robotic systems," IEEE Trans. Syst., Man, Cybern., vol. 21, no. 1, pp. 13 24, Jan./Feb. 1991.

[17] B. J. Stephens and C. G. Atkeson, "Dynamic balance force control for compliant humanoid robots," in Proc. IEEE Conf. Intell. Robots Syst., 2010, pp. 1248-1255.

[18] S.-H. Lee and A. Goswami, "A momentum-based balance controller for humanoid robots on non-level and non-stationary ground," Auton. Robots, vol. 33, no. 4, pp. 399-414, 2012.

[19] L. Righetti, J. Buchli, M. Mistry, and S. Schaal, "Control of legged robots with optimal distribution of contact forces," in Proc. 11th IEEE-RAS Int Conf. Humanoids Robots, 2011, pp. 318-324.

[20] S.-H. Hyon, J. G. Hale, and G. Cheng, "Full-body compliant humanhumanoid interaction: Balancing in the presence of unknown external forces," IEEE Trans. Robot., vol. 23, no. 5, pp. 884-898, Oct. 2007.

[21] W. Kim, J. Lee, N. Tsagarakis, and A. Ajoudani, "A real-time and reducedcomplexity approach to the detection and monitoring of static joint overloading in humans," in Proc. Int. Conf. Rehabil. Robot., 2017, pp. 828-834.

[22] J. Jovic, A. Escande, K. Ayusawa, E. Yoshida, A. Kheddar, and G. Venture, "Humanoid and human inertia parameter identification using hierarchical optimization," IEEE Trans. Robot., vol. 32, no. 3, pp. 726-735, Jun. 2016.

[23] S. H. Hyon, "Compliant terrain adaptation for biped humanoids withou measuring ground surface and contact forces," IEEE Trans. Robot., vol. 25, no. 1, pp. 171-178, Feb. 2009.

[24] H. Jeong, K. Yamada, M. Kido, S. Okada, T. Nomura, and Y. Ohno, "Analysis of difference in center-of-pressure positions between experts and novices during asymmetric lifting," IEEE J. Transl. Eng. Health Med., vol. 4, pp. 1-11, 2016.

[25] A. González, M. Hayashibe, E. Demircan, and P. Fraisse, "Center of mass estimation for rehabilitation in a multi-contact environment: A simulation study," in Proc. IEEE Int. Conf. Syst., Man, Cybern., 2013, pp. 4718-4723.

[26] J. Carpentier, M. Benallegue, N. Mansard, and J.-P. Laumond, "Centerof-mass estimation for a polyarticulated system in contact-A spectral approach," IEEE Trans. Robot., vol. 32, no. 4, pp. 810-822, Aug. 2016.

[27] D. A. Winter, "Human balance and posture control during standing and walking," Gait Posture, vol. 3, pp. 193-214, 1995.

[28] M. B. Popovic, A. Goswami, and H. Herr, "Ground reference points in legged locomotion: Definitions, biological trajectories and control implications," Int. J. Robot. Res., vol. 24, no. 12, pp. 1013-1032, 2005.

[29] P. R. Bélanger, P. Dobrovolny, A. Helmy, and X. Zhang, "Estimation of angular velocity and acceleration from shaft-encoder measurements," Int. J. Robot. Res., vol. 17, no. 11, pp. 1225-1233, 1998.

[30] K. Hornik, "Approximation capabilities of multilayer feedforward networks," Neural Netw., vol. 4, no. 2, pp. 251-257, 1991.

[31] S. Karsoliya, "Approximating number of hidden layer neurons in multiple hidden layer BPNN architecture," Int. J. Eng. Trends Technol., vol. 3, no. 6, pp. 714-717, 2012.

[32] A. Ajoudani et al., "Exploring teleimpedance and tactile feedback for intuitive control of the pisa/IIT soft hand," IEEE Trans. Haptics, vol. 7, no. 2, pp. 203-215, Apr.-Jun. 2014.

[33] J. C. E. Van Der Burg, J. H. Van Dieën, and H. M. Toussaint, "Lifting an unexpectedly heavy object: The effects on low-back loading and balance loss," Clin. Biomechanics, vol. 15, no. 7, pp. 469-477, Aug. 2000. 